\title{
Extraction of Fennel (Foeniculum vulgare) Seeds: Process Optimization and Antioxidant Capacity of the Extracts
}

\author{
G. Angelov* and S. Boyadzhieva \\ Institute of Chemical Engineering, \\ Bulgarian Academy of Sciences, \\ Acad. Bonchev st., Block 103, 1113 Sofia, Bulgaria
}

doi: 10.15255/CABEQ.2015.2281

Original scientific paper

Received: July 20, 2015

Accepted: June 9, 2016

\begin{abstract}
This paper presents the study on the extraction of bioactive substances from fennel seeds. The impact of the main process variables (solvent composition, liquid-to-solid ratio, temperature, contact time) on the concentration of the target substances (polyphenols and flavonoids) in the extracts is studied resulting in the selection of a set of operating parameters, at which their content is maximized. Extracts with higher concentration of target compounds demonstrate higher antioxidant capacity, which confirms the contribution of these substances to better antioxidant performance. The performance of two types of solvents is compared: water and ethanol-water mixtures, showing that water extraction produces more concentrated extracts with higher antioxidant capacity. The extraction kinetics is simulated using Peleg's equation, and a good fit to experimental data is observed. Data for initial extraction rate and equilibrium concentrations is obtained. Based on the combination of experimental and simulation results, an equation is proposed for determination of antioxidant capacity of water extracts of fennel seeds when the phenolic concentration is known, and vice versa - calculation of concentration of polyphenolic compounds in extracts with known antioxidant capacity.
\end{abstract}

Key words:

Foeniculum vulgare, polyphenols, flavonoids, process optimization, antioxidant capacity, Peleg's equation

\section{Introduction}

Antioxidants are substances that protect cells from damage caused by unstable molecules known as free radicals, which induce oxidative stress. The normal cell processes produce free radicals as a by-product, and antioxidants serve to neutralize them. Polyphenolic and flavonoid compounds, which are found in many fruits, vegetables, and tea, are believed to be among the main substances responsible for antioxidant activity ${ }^{1,2}$. This work is focused on estimation of polyphenolic and flavonoid content in fennel (Foeniculum vulgare) seeds, which are traditionally used for medicinal purposes and food flavoring ${ }^{3}$. Although wide studies of the fennel essential oil exist ${ }^{3-5}$, little information is available on its non-volatile constituents. It has been found that fennel distillation wastes possess high antioxidant activity, which has been attributed to non-volatile compounds, mainly phenolic substances ${ }^{6,7}$. Consequently, the antioxidant potential of the fennel plant material might explain some of its uses in folk medicine: for treatment of diabetes, bronchitis, chronic coughs, and kidney stones. Some of these chronic diseases are related to the produc-

"Correspondence: e-mail: gang@bas.bg, fax.:+359 8707523 tion of radical species involved in oxidative stress $^{8}$. Therefore, the quantitative investigation of the content of bioactive ingredients like polyphenols and flavonoids, which are believed to be responsible for the antioxidant capacity of the fennel plant material, is an interesting endeavor and corresponds to the worldwide trends of using natural products as remedies.

In a previous study ${ }^{9}$, the extraction of polyphenols and flavonoids from fennel seeds was studied using water as a solvent. As the extraction capacity depends on the solvent type and its polarity, it is worthy to enhance this study by including comparative tests with other solvents. The general purpose of this work is to examine the extraction capacity of another GRAS (Generally Recognized as Safe) solvent, water-ethanol mixture, on fennel seeds and compare it to the results of water extraction.

The blending of ethanol and water in different proportions results in mixtures with different polarity, i.e. with a different capacity to dissolve target compounds. Thus, an appropriate mixture with maximum affinity to these target substances can be selected. Additionally, this study is focused on process optimization aimed at obtaining enriched extracts with maximum phenolic content and maximum antioxidant capacity (AOC). 


\section{Materials and methods}

\section{Plant material and pretreatment}

Ripe greenish-brown seeds of cultivated fennel (Foeniculum vulgare Mill.) were collected in the East Bulgaria region in 2013. The seeds were dried naturally during storage and their retained humidity was $11 \%$. For the purposes of this study, they were milled and sieved in order to separate a fraction with particle size $0.1-1 \mathrm{~mm}$.

\section{Chemicals}

Folin-Ciocalteu reagent (2 N solution, Sigma), gallic acid (Sigma), dehydrated $\mathrm{Na}_{2} \mathrm{CO}_{3}$ (Valerus), ethanol (96\%, Valerus), $\mathrm{DPPH}^{+}\left(\mathrm{Sigma}^{2}\right.$, methanol (99.9 \% Lab Scan) were used for polyphenol analyses and for determining the antioxidant capacity of the extracts. Quercetin and other chemicals necessary for flavonoid analyses were supplied from Sigma-Aldrich.

\section{Analyses}

\section{Total phenolic content}

The total phenolic content of fennel seed extracts was determined spectrophotometrically with FolinCiocalteu reagent. An aliquot of the extract $(0.02$ $\mathrm{mL}$ ) was mixed with $0.1 \mathrm{~mL}$ of $2 \mathrm{~N}$ Folin-Ciocalteu reagent and $0.3 \mathrm{~mL}$ of $\mathrm{Na}_{2} \mathrm{CO}_{3}(20 \% \mathrm{w} / \mathrm{v})$, all diluted to $2 \mathrm{~mL}$ with water. The resulting mixture was incubated at room temperature for 2 hours for color development. The absorbance of the samples was measured at $765 \mathrm{~nm}$ using double beam UV/VIS spectrophotometer UNICAM $\AA$-Helios $\beta$. Calibration line with gallic acid was made, and the total phenolic content was expressed as gallic acid equivalent ${ }^{10,11}$. The reference cuvette contained all reagents except the extract sample.

\section{Total flavonoids content}

The analytical method for flavonoids is based on formation of chemical complex flavonoids - aluminium. Two $\mathrm{mL}$ of $2 \% \mathrm{AlCl}_{3}$ ethanol solution was added to $2 \mathrm{~mL}$ of analyzed liquid extract. After one hour of incubation at room temperature for color development, the absorbance was measured at $420 \mathrm{~nm}$ using UV-VIS spectrophotometer. The results were expressed as quercetin equivalent according to a quercetin calibration curve ${ }^{12}$.

\section{Antioxidant capacity (AOC)}

AOC is determined by the DPPH method, which is largely used because of its simplicity and reproducible results ${ }^{13,14}$. This method is based on the reaction of antioxidant substances with methanol solution of DPPH, resulting in neutralization of free radicals emitted by DPPH. The latter absorbs at 517 $\mathrm{nm}$, but upon reduction by an antioxidant the absorption decreases, and the color changes from deep violet to yellow. The absorption is measured spectrophotometrically.

The analytical protocol is described below:

The blank sample is adjusted by measuring a cuvette with a mixture of $1 \mathrm{~mL}$ solvent and $4 \mathrm{~mL}$ methanol solution of DPPH against methanol cuvette $\left(A_{0}\right)$. The analyzed sample is obtained by mixing $1 \mathrm{~mL}$ of plant extract with $4 \mathrm{~mL} 0.004 \%(0.1$ $\mathrm{mM}$ ) solution of DPPH in methanol. After $60 \mathrm{~min}$ incubation in darkness, the light absorbance of the sample is measured against methanol cuvette at 517 $\mathrm{nm}\left(A_{\mathrm{s}}\right)$. The inhibition capacity (IC) of the sample is calculated by the expression:

$$
\mathrm{IC}[\%]=\left(1-A_{\mathrm{s}} / A_{0}\right) \cdot 100
$$

The antioxidant capacity is expressed as IC50 value, which represents the concentration of a sample that inhibits $50 \%$ of the free radicals added to the system. IC50 value can be determined from the chart that expresses IC as a function of the extract concentration $C_{\mathrm{s}}$.

The graphical relationship IC $=\mathrm{f}\left(C_{\mathrm{s}}\right)$ for an extract is obtained by measuring the absorption of a series of samples containing different amounts of this extract added to the solvent [ $\left.\mathrm{mL} \mathrm{L}^{-1}\right]$. Appropriate dilution of the samples is necessary in order to fall in the linear part of the graph in IC interval 0 to above $50 \%$. The extract concentration reducing 50 $\%$ of free radicals can be calculated from the linear equation by setting $\mathrm{IC}=50$, or determined from the chart as the abscissa of the intersection point of the horizontal line from the $50 \%$ IC ordinate and the data line. A smaller value of $C_{\mathrm{s}}$ corresponds to higher AOC, i.e. a smaller quantity of this extract is needed for neutralization of $50 \%$ of the free radicals. IC50 concentration can be transformed and expressed as mg DPPH neutralized by $1 \mathrm{~g}$ of dry extract [mg DPPH $\mathrm{g}^{-1} \mathrm{de}$ ] or $1 \mathrm{~g}$ of raw material (rm) $\left[\mathrm{mg} \mathrm{DPPH} \mathrm{g}^{-1} \mathrm{rm}\right]$.

\section{Dry matter yield}

After extraction, $10 \mathrm{~mL}$ samples of the liquid extract were dried at $80{ }^{\circ} \mathrm{C}$ until constant weight was reached (henceforth referred to as dry extract - de). Laboratory analytical balance Sartorius with $0.1 \mathrm{mg}$ accuracy was used.

\section{Experimental}

The present study is focused on the identification of optimal operating conditions, specifically: 1) suitable solvent, 2) minimum liquid-to-solid ratio necessary to avoid solubility limitations, 3 ) operat- 
ing temperature, and 4) duration of the extraction process. The range of variation of these parameters is:

- Solvent concentration: 0 - $96 \%$ ethanol;

- Liquid-to-solid ratio: 5 - 20;

- Temperature: $20-70{ }^{\circ} \mathrm{C}$;

- Time: 3 - 150 minutes.

The extraction yield and the antioxidant activity of plant extracts are highly dependent on the solvent polarity. The highest yields are usually achieved with ethanol and methanol, and their mixtures with water, although other solvents, such as ethyl acetate or acetone, have also been used for extraction of polyphenols from plants ${ }^{15}$. Water and ethanol are the most widely used because of their low toxicity and high extraction yields. These solvents are suitable for extraction of phenolic compounds, most of which are also of polar type. Additionally, the resulting polarity of water-ethanol mixtures varies depending on the proportion of solvent constituents, which might be favorable for dissolution of phenolic compounds with different polarity. Therefore, harmless polar solvents (water, ethanol, and their mixtures) have been chosen for the experiments.

\section{Preparation and sampling of extracts}

All extracts were prepared by mixing $5 \mathrm{~g}$ of ground plant material (further referred to as raw material - rm) with a corresponding amount of solvent, which provides a specified value of solvent-to-solid ratio. The extractions were carried out in a thermostatic water bath shaker (New Brunswick Scientific) at $160 \mathrm{rpm}$. In order to attain pseudo-equilibrium, long contact time was applied (2 hours), after which the liquid phase was sampled and analyzed. In case of kinetic experiments, parallel extraction of a number of identical mixtures was carried out, each one being sampled and analyzed at different times, in order to determine the concentration evolution in the course of time. The results were represented as the mean value of 2-3 parallel samples, and standard deviations were calculated. In addition, statistical analysis of the results was performed (ANOVA test, f-test, k-test) using Microsoft software.

\section{Modeling}

In this work, Peleg's model was used ${ }^{16}$. It was initially introduced for description of sorption curves (moisture content depending on time). It could be also applied to extraction kinetics curves (extracted substance over time), because both curves have a similar asymptotic shape.

Peleg's equation reads:

$$
C(t)=C_{0}+\frac{t}{K_{1} t+K_{2}}
$$

$C(t)$ is the concentration of extracted substance after time $t, K_{1}$ and $K_{2}$ are constants, $C_{0}$ is the concentration of extracted substance at the initial time $t$ $=0$. As the value of $C_{0}$ is zero, Eq. (1) is reduced to:

$$
C(t)=\frac{t}{K_{1} t+K_{2}}
$$

The extraction rate in the time $t$ can be obtained by differentiation of (3)

$$
\frac{\mathrm{d} C(t)}{\mathrm{d} t}=\frac{K_{2}}{\left(K_{1} t+K_{2}\right)^{2}}
$$

At time $t=0$, Eq. (4) takes the form:

$$
\frac{\mathrm{d} C(0)}{\mathrm{d} t}=\frac{1}{K_{2}}=R_{0}
$$

So, the physical meaning of $K_{1}$ is related to the initial extraction rate $R_{0}$.

When $t \rightarrow \infty$, i.e. at equilibrium state, Eq. (3) becomes

$$
C(t)_{\mid t \rightarrow \infty}=C_{\mathrm{e}}=\frac{1}{K_{1}}
$$

Thus, the constant $K_{2}$ is related to the concentration at equilibrium state $C_{\mathrm{e}}$.

Eq. (3) can be arranged in linear form

$$
\frac{t}{C(t)}=K_{1} t+K_{2}
$$

As seen, $K_{1}$ and $K_{2}$ can be determined from the slope and the intercept of the straight line representing this equation.

\section{Results and discussion}

\section{Extraction with ethanol-water mixtures}

Determination of appropriate solvent concentration

Fig.1a illustrates the effect of different water-ethanol mixtures on the total extract yield defined as mg of extracted mass per $\mathrm{g}$ of raw material. The yield of polyphenols and flavonoids [mg of corresponding substance $\mathrm{g}^{-1} \mathrm{rm}$ ] is presented in Fig. 1b. Supposing better solubility, a higher process temperature $\left(70{ }^{\circ} \mathrm{C}\right)$ close to the ethanol boiling point was chosen for these runs. The value of liquid-to-solid ratio (hydromodule) was also high $(15: 1)$ in order to ensure an abundant amount of solvent. It was supposed that at these excessive conditions (high temperature, high hydromodule, and long contact time $2 \mathrm{~h}$ ) the yield should be maximized. 


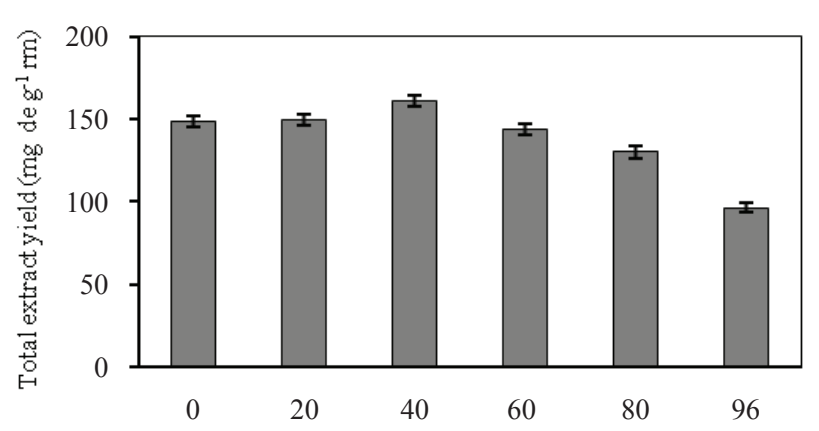

a)

Conc. $\operatorname{EtOH}(\%)$

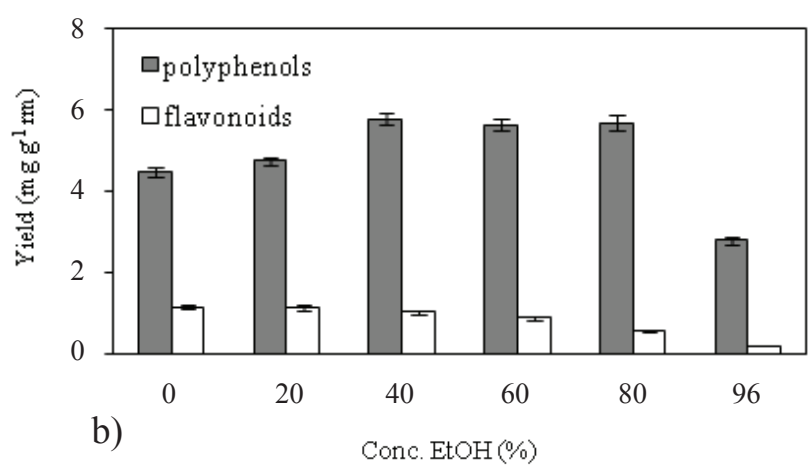

Fig. 1 - Influence of solvent composition on the yield. Hydromodule 15 , contact time $2 \mathrm{~h}, \mathrm{~T}=70^{\circ} \mathrm{C}$, shaker at $160 \mathrm{rpm}$

a) - total extract yield; b) yield of polyphenols and flavonoids.

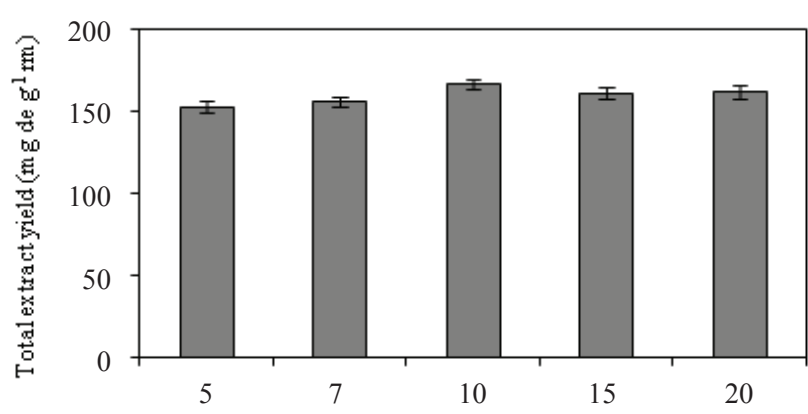

a)

Hydrom odule

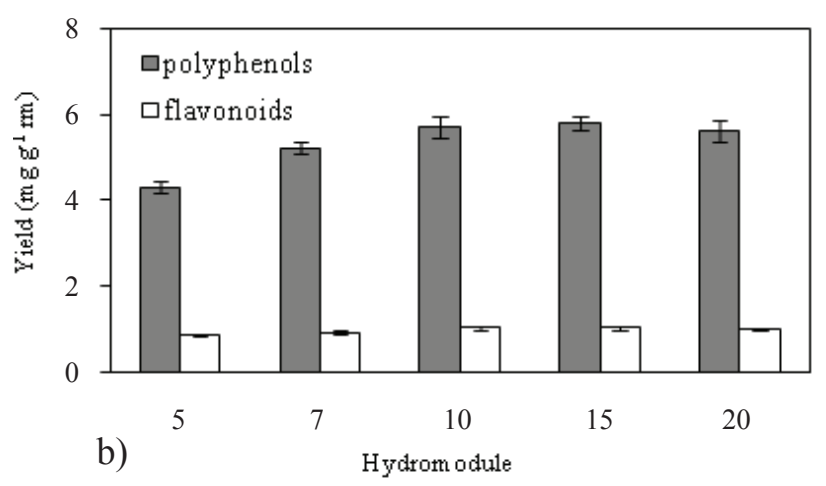

Fig. 2 - Yield at different hydromodules. $40 \%$ ethanol, contact time $2 \mathrm{~h}, T=70^{\circ} \mathrm{C}$, shaker at $160 \mathrm{rpm}$ a) dry extract; b) polyphenols and flavonoids.
By visual inspection of the charts, the total extract yield attains its maximum with $40 \%$ ethanol. The lowest quantity is extracted with $96 \%$ ethanol, and the yield drops with proportions of ethanol below $40 \%$ (Fig. 1a).

Concerning polyphenols (Fig. 1b), the yield stays at its maximum in the interval $40-80 \%$ ethanol. Solvents containing 0 to $40 \%$ ethanol still perform better than those containing $96 \%$ ethanol.

The content of flavonoids is about $5-6$ times lesser than that of polyphenols. Their yield is reduced when increasing ethanol concentration (Fig. 1b).

The above observations are supported by the results of single factor ANOVA tests with significance level 0.05. Statistically, similar (not significantly different) yields of flavonoids and dry extracts were obtained with $0-60 \%$ ethanol, while the polyphenol yields were similar for extractions with $40-80 \%$ ethanol.

Combining the statistical estimations with the quantitative results of Fig. 1, it can be concluded that the best extraction of both polyphenols and flavonoids at lower price of the solvent is obtained with $40 \%$ ethanol. This value appears to be the optimal concentration allowing for extraction of both water-soluble and ethanol-soluble antioxidant substances. Consequently, further experiments were carried out with this composition of the solvent.

\section{Selection of solvent-to-solid ratio (hydromodule)}

In order to ensure operation with minimum but sufficient quantity of solvent, runs at different hydromodules were carried out (Fig. 2). High temperature and long contact time were applied in order to improve solubility and attain pseudo-equilibrium.

As seen from Fig. 2, the increase in solvent quantity up to hydromodule 10 leads to increased amounts of extracted substances in terms of total extract and polyphenols, i.e. at hydromodule less than 10 , the solvent quantity is insufficient for a complete extraction. At hydromodule 10 or higher, there are no significant changes in the extracted quantity, i.e. more solvent does not extract additional matter from the solid. Flavonoid extract yields do not visually appear to have strong correlation to hydromodule value (Fig. 2b).

The ANOVA tests confirm the visual observations and show insignificant impact of hydromodule on the yields of flavonoids in the entire range of variation of this parameter, as well as insignificant differences in the yield of polyphenols and total extract at hydromodule 10 or higher.

Since the dependence on hydromodule is not clearly seen in the case of flavonoids (Fig. 2b), and 
in view of their minor concentrations and smaller contribution to the properties of the extracts, it seems better to select a hydromodule value optimized for extraction of components presented in high quantity.

Therefore, liquid-to-solid ratio of 10:1 was selected, and all further experiments were conducted at this ratio.

\section{Selection of extraction temperature}

Fig. 3 illustrates the yields at different temperatures along with the antioxidant capacity of the extracts.

The yield at higher temperature is higher (Fig. $3 a, b)$, which in turn results in a higher antioxidant
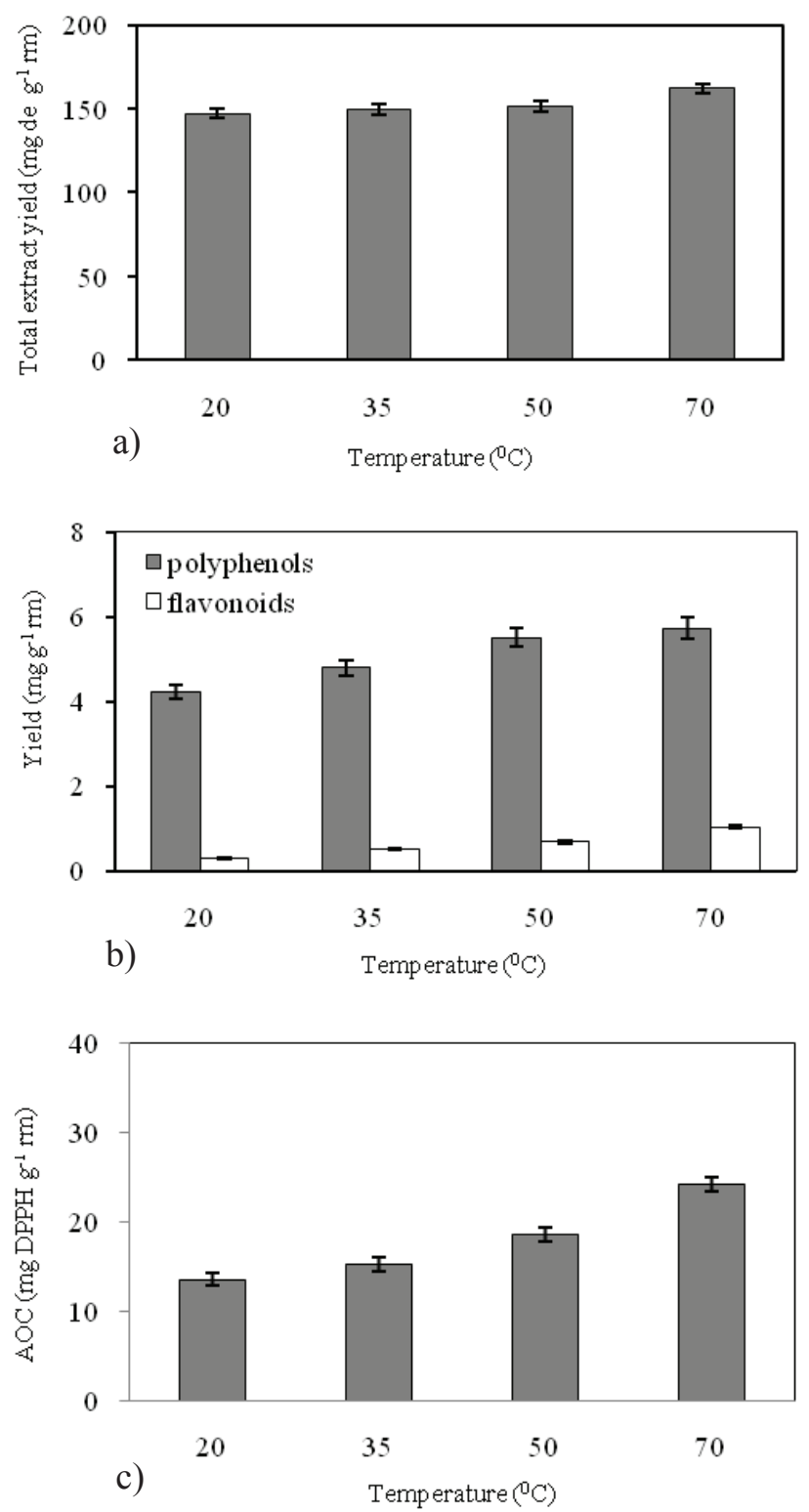

Fig. 3 - Yield $(a, b)$ and antioxidant capacity (c) of extracts obtained at different temperature, $40 \%$ ethanol, hydromodule 10:1, 2 hours extraction in shaker at $160 \mathrm{rpm}$ capacity (Fig. 3c). No yield reduction is observed when raising the temperature from 20 to $70{ }^{\circ} \mathrm{C}$, which is an indication that the active ingredients are thermo-stable at these temperatures.

The results of statistical tests for the temperature effects are:

- For flavonoids and AOC - significant impact in the entire interval $20-70{ }^{\circ} \mathrm{C}$;

- For polyphenols - significant impact in the interval $20-50{ }^{\circ} \mathrm{C}$, insignificant impact in the interval $50-70{ }^{\circ} \mathrm{C}$.

- For dry extract - insignificant impact in the interval $20-50{ }^{\circ} \mathrm{C}$, significant impact in the interval $50-70{ }^{\circ} \mathrm{C}$.

Based on the above results, temperature $70{ }^{\circ} \mathrm{C}$ should be chosen for production of extracts with highest antioxidant capacity.

\section{Process kinetics and minimum contact time}

Figs. $4(\mathrm{a}, \mathrm{b})$ illustrate the development of extraction process over time along with the antioxidant capacity of the corresponding extracts (Fig. 4c).

The range of deviation of results in Fig. 4 from their mean values was $1.5-7.5 \%$. Typically, higher deviation was registered when measuring small values.

Generally, the extraction process can be divided into three periods, which are illustrated by the curves for total extract and polyphenols. The initial period (about $10 \mathrm{~min}$ ) of fast mass transfer and fast rising yield corresponds to dissolution of easily available substances located on the particles' surface. The next period of decelerating mass transfer rate (from 10 to about $90 \mathrm{~min}$ ) reflects the simultaneous dissolution of residual extractable substances from the surface and from the interior of the particles. The last slowly increasing part corresponds to mass transfer from internal pores. Over time, the yield asymptotically approaches pseudo-equilibrium state (plateau).

According to the results of statistical analysis, the yield of flavonoids becomes similar at contact time in the range $60-150 \mathrm{~min}$, i.e. the extraction of these compounds is practically completed in 60 minutes. For polyphenols and dry extract, the productive contact time is $90 \mathrm{~min}$, i.e. contact time longer than 90 minutes does not increase significantly the yield of any substance. Analogously, AOC attains its highest value at $90 \mathrm{~min}$, becoming statistically similar afterwards.

Consequently, it might be concluded that pseudo-equilibrium state is attained after about 90 minutes, and this process duration can be selected as the shortest and optimal contact time necessary for completing the extraction. 

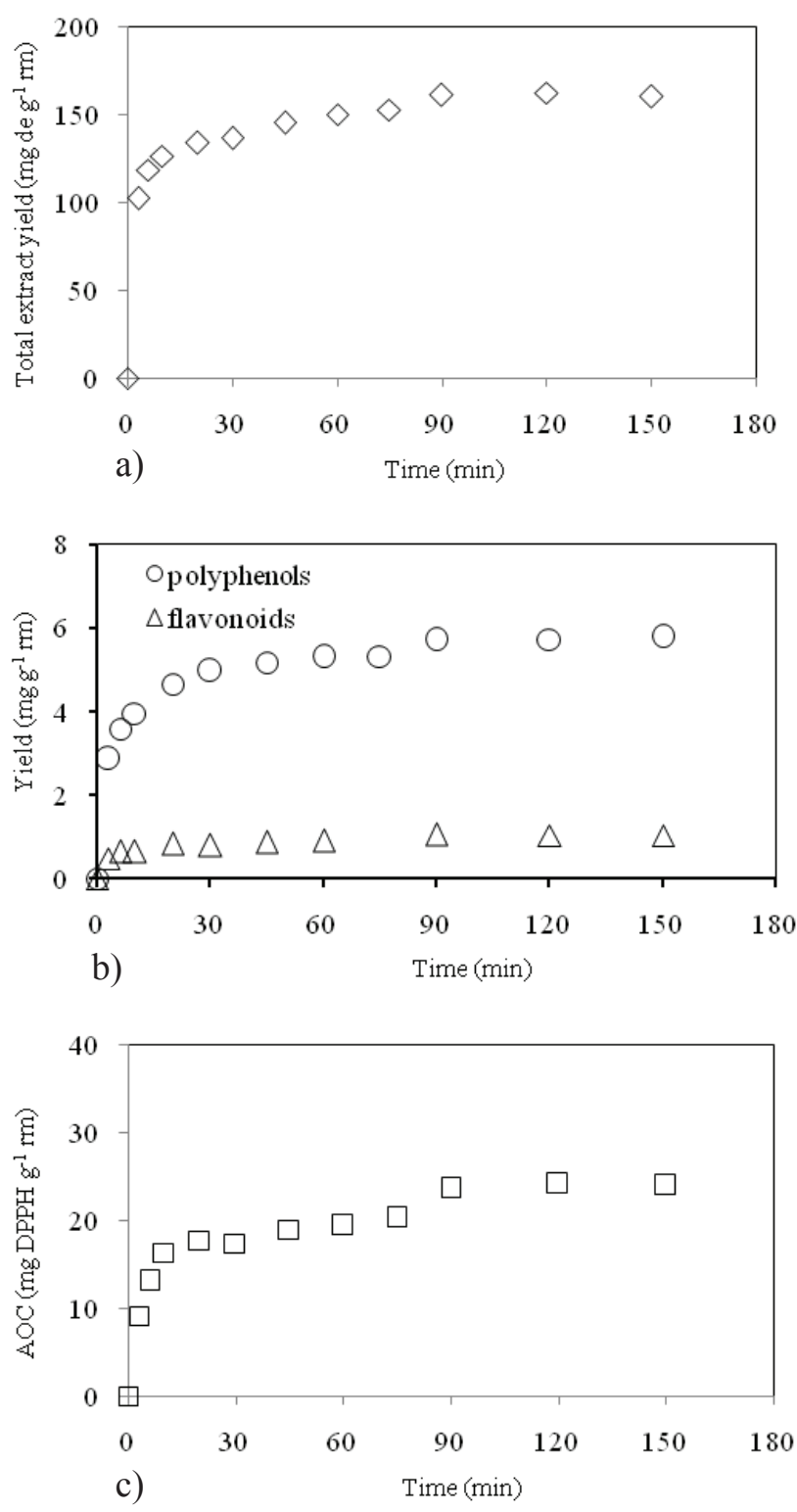

Fig. 4 - Extraction kinetics and antioxidant capacity of extracts obtained with $40 \%$ ethanol, temperature $70^{\circ} \mathrm{C}$, hydromodule 10:1, extraction in shaker at $160 \mathrm{rpm}$

$a$ - total extract, $b$ - polyphenols and flavonoids, $c$ - antioxidant capacity

\section{Water extraction of fennel}

As seen from Fig. 1a, the total mass extracted with water is not very far from that obtained with $40 \%$ ethanol. Presuming that water extraction might be cheaper at comparable efficiency, these two solvents are compared in more details below. The results for water extraction of polyphenols and flavonoids are taken from a previous study ${ }^{9}$. They are enhanced with data for the antioxidant capacities (AOC) of water extracts.

The impact of temperature on the water extraction yields is similar to that of ethanolic extraction. In Table 1 , data for $70{ }^{\circ} \mathrm{C}$ and $100{ }^{\circ} \mathrm{C}$ are compared. Processing at higher temperatures improves the yield of total extract and polyphenols, as well as AOC of the extracts. Consequently, extraction with boiling water is recommended because it enables obtaining more extracted mass with higher antioxidant capacity.

Unlike $40 \%$ ethanol extraction (hydromodule 10:1), the appropriate hydromodule for water extraction has been determined to be $20: 1 .^{9}$ It means that twice more solvent (water) has to be heated for the extraction itself, and more water has to be evaporated at a later stage for obtaining dry extract, i.e. the process is more energy-consuming. However, the solvent is cheaper, safe, and non-flammable.

The appropriate process duration has been determined by observing the yields over time ${ }^{9}$. Steadystate is attained after about 60 minutes, which is shorter than the process duration for $40 \%$ ethanol (90 minutes).

\section{Comparison of results for extraction with water and $40 \%$ ethanol}

The results in Table 1 allow for comparison of both studied processes:

- The yield of total extract and bioactive components is improved at higher temperature.

- The extracted mass is $16-18 \%$ of the mass of raw material.

Table 1 - Experimental pseudo-equilibrium concentrations (120 minutes contact time)

\begin{tabular}{|c|c|c|c|c|c|c|c|c|}
\hline \multirow{2}{*}{ Solvent } & \multirow{2}{*}{\begin{tabular}{|c|} 
Temp. \\
${ }^{0} \mathrm{C}$ \\
\end{tabular}} & \multicolumn{2}{|c|}{ Yield $\mathrm{Pph}^{1}$} & \multicolumn{2}{|c|}{ Yield $\mathrm{Fl}^{2}$} & \multicolumn{2}{|c|}{$\mathrm{AOC}^{3}$} & \multirow{2}{*}{\begin{tabular}{|c} 
Total extract yield \\
${\mathrm{mg} \mathrm{de} \mathrm{g}^{-1} \mathrm{rm}}^{2}$
\end{tabular}} \\
\hline & & $\mathrm{mg} \mathrm{g}^{-1} \mathrm{rm}^{4}$ & $m g g^{-1} \mathrm{de}^{5}$ & $\mathrm{mg} \mathrm{g}^{-1} \mathrm{rm}$ & $\mathrm{mg} \mathrm{g}^{-1} \mathrm{de}$ & mg DPPH g-1 $\mathrm{g}^{-1}$ & mg DPPH $g^{-1} \mathrm{de}$ & \\
\hline $\begin{array}{l}40 \% \text { ethanol } \\
\left(\mathrm{L}: \mathrm{S}^{6}=10: 1\right)\end{array}$ & 50 & $5.5 \pm 0.16$ & $36.2 \pm 1.23$ & $0.7 \pm 0.03$ & $4.5 \pm 0.18$ & $18.2 \pm 0.75$ & $4.5 \pm 0.18$ & $151.8 \pm 2.66$ \\
\hline $\begin{array}{l}40 \% \text { ethanol } \\
(\mathrm{L}: \mathrm{S}=10: 1)\end{array}$ & 70 & $5.7 \pm 0.13$ & $35.2 \pm 1.02$ & $1.0 \pm 0.04$ & $6.4 \pm 0.16$ & $24.1 \pm 0.79$ & $6.4 \pm 0.16$ & $162.3 \pm 2.29$ \\
\hline $\begin{array}{l}\text { Water* } \\
(\mathrm{L}: S=20: 1)\end{array}$ & 70 & 7.5 & 44.6 & 1.6 & 9.7 & 31.1 & 9.7 & 168.2 \\
\hline $\begin{array}{l}\text { Water* } \\
(L: S=20: 1)\end{array}$ & 100 & 8.2 & 46.4 & 1.8 & 9.0 & 40.9 & 9.0 & 177.1 \\
\hline
\end{tabular}

${ }^{1} \mathrm{Pph}$ - polyphenols; ${ }^{2} \mathrm{Fl}$ - flavonoids; ${ }^{3} \mathrm{AOC}$ - antioxidant capacity; ${ }^{4} \mathrm{rm}$ - raw material; ${ }^{5} \mathrm{de}$ - dry extract; ${ }^{6} \mathrm{~L}: \mathrm{S}$ - liquid-to-solid ratio; *Data for water extraction are taken from [9]. 


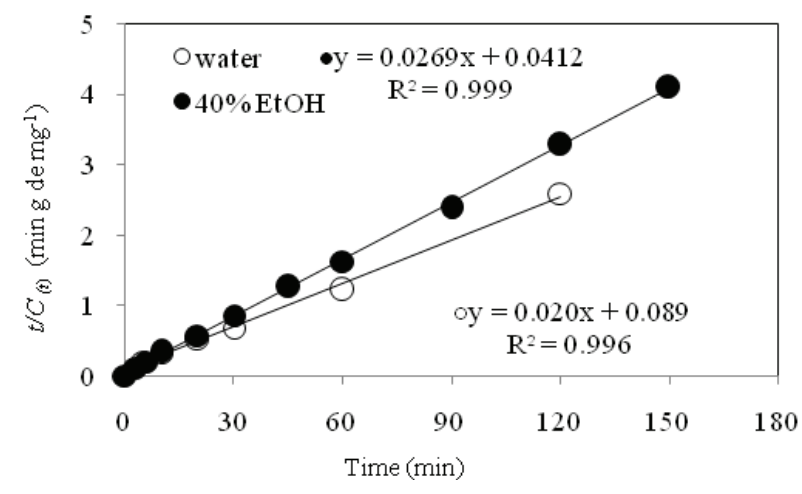

Fig. 5 - Eq. (7) applied to extraction of polyphenols with different solvents (water at $100{ }^{\circ} \mathrm{C}$ and $40 \%$ ethanol at $\left.70{ }^{\circ} \mathrm{C}\right)$

- The yield of polyphenols, flavonoids, and total dry extract is higher when using water as a solvent.

- The water extracts of fennel have higher antioxidant capacity, which might be attributed to the higher content of polyphenols.

It should be reminded that water extraction demands twice more solvent and higher processing temperature than ethanol-water extraction. It means that more energy will be consumed for solvent heating and evaporation in the production of dry extract. On the other hand, the contact time is shorter (60 min vs $90 \mathrm{~min}$ ) and the solvent (water) is cheap and safe (fire and explosion proof). The final choice of solvent should be made on the basis of economic calculations.

\section{Peleg's model applied to experimental process kinetics}

The parameters of Peleg's equation (constants $K_{1}$ and $K_{2}$ ) can be obtained by plotting the left side of Eq. (7) $t / C(t)$ vs. time. Fig. 5 illustrates the case of polyphenols extraction.

It is seen that the experimental results for the concentration of extracted polyphenols over time match fairly well a linear dependence as prescribed by Eq. (7). Strong linearity was registered also with the experimental kinetic results for total extracted matter and extracted flavonoids $\left(R^{2}=0.990-\right.$ 0.999). Consequently, the obtained linear equations may be used for determination of model parameters $K_{1}$ and $K_{2}$, which characterize the equilibrium concentration $C_{\mathrm{e}}$ and the initial extraction rate $R_{0}$. The results are summarized in Table 2. According to these results, higher values for equilibrium concentrations are obtained by water extraction, i.e. Peleg's model states that water is more efficient as a solvent, as it is observed experimentally, as well.

Comparing the results from Table 1 and Table 2 , it becomes clear that Peleg's equation systemati-
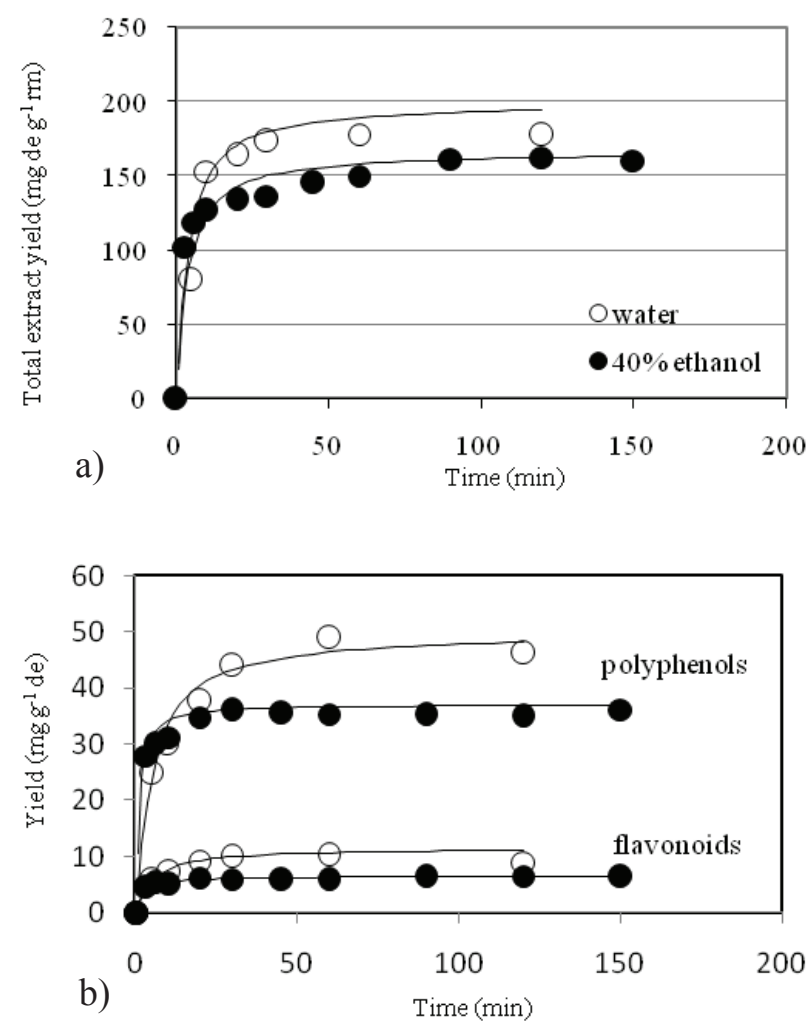

Fig. 6 - Comparison of model and experimental extraction kinetics of total extract (a) polyphenols and flavonoids (b). Points: experimental data; lines: calculation by Peleg's equation.

cally stipulates higher values for equilibrium concentrations (Table 2) than these obtained experimentally (Table 1). The difference can be explained by the fact that, in practice, the extraction process is not carried out until its equilibrium state, which will be reached after a long time. The process is usually stopped at a pseudo-equilibrium state, when the yield does not rise significantly at further processing.

Fig. 6 represents kinetic curves obtained by simulation through Peleg's equation compared to experimental results for the real process kinetics, and a good fit is observed.

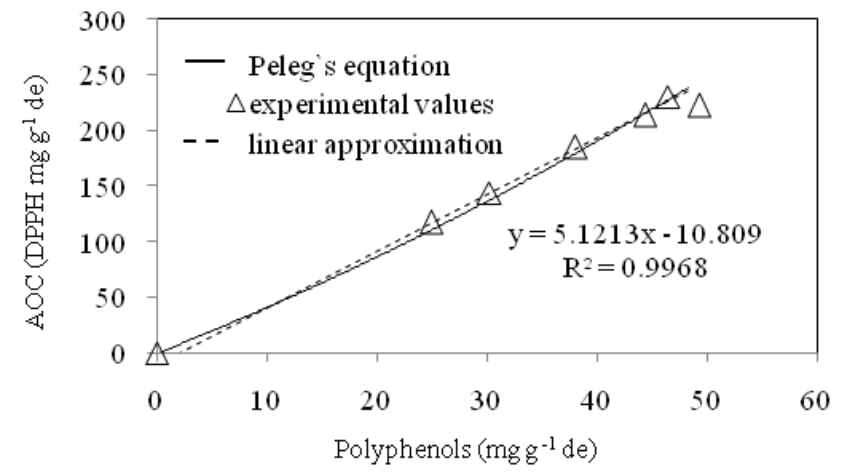

Fig. 7 - Correlation between polyphenols content and AOC of water extracts 
Table 2 - Values of equilibrium concentration $C_{e}$ and initial extraction rate $R_{0}$

\begin{tabular}{|c|c|c|c|c|c|}
\hline \multirow{3}{*}{ Solvent } & \multicolumn{2}{|c|}{ Polyphenols } & \multicolumn{2}{|c|}{ Flavonoids } & Total extract \\
\hline & \multicolumn{5}{|c|}{ Model equilibrium concentration $C_{\mathrm{e}}=1 / K_{1}$} \\
\hline & $\mathrm{mg} \min \mathrm{g}^{-1} \mathrm{rm}^{1}$ & $\operatorname{mg} \min \mathrm{g}^{-1} \mathrm{de}^{2}$ & $\mathrm{mg} \min \mathrm{g}^{-1} \mathrm{rm}$ & $\operatorname{mg} \min \mathrm{g}^{-1} \mathrm{de}$ & $\mathrm{mg}$ de $\min \mathrm{g}^{-1} \mathrm{rm}$ \\
\hline $\begin{array}{l}40 \% \text { ethanol } \\
\mathrm{L}: \mathrm{S}^{3}=10 ; 70{ }^{\circ} \mathrm{C}\end{array}$ & 5.9 & 37.0 & 1.1 & 6.5 & 166.6 \\
\hline \multirow[t]{3}{*}{$\begin{array}{l}\text { Water } \\
\mathrm{L}: \mathrm{S}=20 ; 100{ }^{\circ} \mathrm{C}\end{array}$} & 8.9 & 50.0 & 2.0 & 11.5 & 200.0 \\
\hline & \multicolumn{5}{|c|}{ Initial extraction rate $R_{0}=1 / K_{2}$} \\
\hline & $\mathrm{mg} \mathrm{g}^{-1} \mathrm{rm}$ & $\mathrm{mg} \mathrm{g}^{-1} \mathrm{de}$ & $\mathrm{mg} \mathrm{g}^{-1} \mathrm{rm}$ & $\mathrm{mg} \mathrm{g}^{-1} \mathrm{de}$ & $\mathrm{mg}$ de $\mathrm{g}^{-1} \mathrm{rm}$ \\
\hline $\begin{array}{l}40 \% \text { ethanol } \\
\mathrm{L}: \mathrm{S}=10,70^{\circ} \mathrm{C}\end{array}$ & 1.4 & 30.3 & 0.2 & 2.6 & 50.0 \\
\hline $\begin{array}{l}\text { Water } \\
\mathrm{L}: \mathrm{S}=20 ; 100{ }^{\circ} \mathrm{C}\end{array}$ & 1.2 & 11.2 & 0.5 & 2.5 & 62.5 \\
\hline
\end{tabular}

${ }^{1} \mathrm{rm}$ - raw material; ${ }^{2} \mathrm{de}$ - dry extract; ${ }^{3} \mathrm{~L}: \mathrm{S}$ - liquid-to-solid ratio

In Fig. 7 the antioxidant activity of water extracts is plotted against their phenolic content.

The line is obtained using Peleg's equation, while the points represent experimental values, and a good fit is observed. As seen, this graph appears to approximate a straight line. Thus, a linear approximation can supply a simple equation for determination of AOC of water extracts of fennel seeds when polyphenols concentration $(C \mathrm{pph})$ is known, and vice versa. The dashed line represents this linear approximation based on experimental data obtained in this work and values calculated by Peleg's equation in the interval $0-50 \mathrm{pph}$ units and $0-230$ AOC units. The $R$-squared value of this line is $R^{2}=$ 0.996 and its equation is

$$
\mathrm{AOC}=5.12 \cdot \mathrm{Cpph}-10.81
$$

or

$$
\mathrm{Cpph}=0.20 \cdot \mathrm{AOC}+2.11
$$

It has to be pointed out that, in the case of extraction with ethanol-water mixtures, no grouping along a linear correlation was observed between AOC and polyphenolic content of extracts.

\section{Conclusion}

The subject of this paper is the determination of optimal conditions for batch solvent extraction of fennel seeds in order to obtain extracts with maximum content of antioxidant compounds, namely polyphenols and flavonoids. The impact of the main process parameters (solvent composition, liquid-to-solid ratio, temperature, contact time) on the concentration of the target substances in the extracts is studied, resulting in the selection of a set of operating parameters at which maximum yield is ob- tained. The comparison of ethanol-water extraction with water extraction shows that the latter produces more concentrated extracts with higher antioxidant capacity. The process kinetics of both water and ethanol-water extraction are successfully simulated by Peleg's equation, which enables the determination of the initial extraction rate and equilibrium concentration. It was observed that, unlike ethanolic extracts, water extracts have a strong Linear correlation between polyphenolic content and antioxidant capacity, and an equation is proposed for calculation of one term when the other term is known.

\section{References}

1. Gross, M., Flavonoids and cardiovascular disease, Pharm. Biol. 42 (2004) 21. doi: http://dx.doi.org/10.3109/13880200490893483

2. El Babili, F., Babili, M. E. L., Souchard, J. P., Chatelain, $C$., Culinary decoctions: Spectrophotometric determination of various polyphenols coupled with their antioxidant activities, Pharm. Crop. 4 (2013) 15. doi: http://dx.doi.org/10.2174/2210290601304010015

3. Garcia-Jimenez, N., Perez-Alonso, M. J., Velasco-Negueruela, A., Chemical composition of fennel oil, Foeniculum vulgare Mill. from Spain, J. Essen. Oil. 12 (2000) 159. doi:10.1080/10412905.2000.9699487

4. Moghtader, M., Comparative survey on the essential oil composition from the seeds and flowers of Foeniculum vulgare Mill. from Kerman province, J. Hortic. Forestry 5 (2013) 37. doi: 10.5897/JHF2013.0289

5. Guillén, M. D., Manzanos, M. J., A study of several parts of the plant Foeniculum vulgare as a source of compounds with industrial interest, Food Rev. Int. 29 (1996) 85. doi: http://dx.doi.org/10.1016/0963-9969(95)00047-X

6. Parejo, I., Viladomat, F., Bastida, J., Rosas-Romero, A., Flerlage, N., Burillo, J., Codina, C., Comparison between the radical scavenging activity and antioxidant activity of six distilled and nondistilled Mediterranean herbs and aromatic plants, J. Agric. Food Chem. 50 (2002) 6882. doi: http://dx.doi.org/10.1021/jf020540a 
7. Parejo, I., Jauregui, O., Saänchez-Rabaneda, F., Viladomat, F., Bastida, J., Codina, C., Separation and characterization of phenolic compounds in fennel (Foeniculum vulgare) using liquid chromatography-negative electrospray ionization tandem mass spectrometry, J. Agric. Food Chem. 52 (2004) 3679 .

doi: http://dx.doi.org/10.1021/jf030813h

8. Barros, L., Heleno, S., Carvalho A., Ferreira, I., Systematic evaluation of the antioxidant potential of different parts of Foeniculum vulgare Mill. from Portugal, Food and Chemical Toxicology 47 (2009) 2458. doi: http://dx.doi.org/10.1016/j.fct.2009.07.003

9. Boyadzhieva, S., Angelov, G., Optimization of water extraction of fennel seeds, J. Chem. Technol. and Metallurgy 49 (2014) 447.

10. Singleton, $V$. L., Rossi J. A., Colorimetry of total phenolics with phosphomolybdic-phosphotungstic acid reagents, Am. J. of Enol. Vitic. 16 (1965) 144.

11. Waterhouse, A., Folin-Ciocalteau micro method for total phenol in wine, http://waterhouse.ucdavis.edu/faqs/folin-ciocalteau-micro-method-for-total-phenol-in-wine (22.02.2015)
12. Ordonez, E. Z., Gomez, J. D., Vattuone, M. A., Isla, M. I., Antioxidant activities of Sechium edule (Jacq) swartz extracts, Food Chem. 97 (2006) 452. doi: http://dx.doi.org/10.1016/j.foodchem.2005.05.024

13. Brand-Williams, W., Cuvelier, M. E., Berset, C., Use of a free radical method to evaluate antioxidant activity, Lebensm. Wiss. Technol. 28 (1995) 25. doi: http://dx.doi.org/10.1016/S0023-6438(95)80008-5

14. Sanchez-Moreno, C., Methods used to evaluate the free radical scavenging activity in foods and biological systems, Food Sci. and Technol. Intern. 8 (2002) 121 doi: http://dx.doi.org/10.1177/1082013202008003770

15. Perez-Jimenez, J., Arranz, S., Tabernero, M., Diaz-Rubio M., Serrano, J., Gono, I., Saura-Calixto, F., Updated methodology to determine antioxidant capacity in plant foods, oils and beverages: Extraction, measurement and expression of results, Food Research Intern. 41 (2008) 274. doi: http://dx.doi.org/10.1016/j.foodres.2007.12.004

16. Peleg, M., An empirical model for the description of moisture sorption curves, J. Food Sci. 53 (1988) 1216. doi: http://dx.doi.org/10.1111/j.1365-2621.1988.tb13565.x4 